\title{
Cloreto de sódio, benzocaína e óleo de cravo-da-índia na água de transporte de tilápia-do-nilo
}

\author{
José Ricardo Oliveira ${ }^{1}$, João Laurindo do Carmo ${ }^{2}$, Karine Kelly Cavalcanti Oliveira ${ }^{3}$, \\ Maria do Carmo Figueredo Soares ${ }^{2}$
}

\author{
${ }^{1}$ Escola Agrotécnica Federal de Vitória de Santo Antão - PE (EAFVS-PE). \\ 2 Departamento de Pesca e Aquicultura - UFRPE. \\ 3 Universidade Federal Rural de Pernambuco - UFRPE.
}

RESUMO - Testou-se a ação de diferentes concentrações de cloreto de sódio, benzocaína e óleo de cravo-da-índia na sobrevivência de alevinos e juvenis de tilápia (Oreochromis niloticus), linhagem chitralada, por 5 horas visando ao transporte. Utilizaram-se 1.350 alevinos $(9,74 \pm 0,04 \mathrm{~g}$ e $6,79 \pm 0,01 \mathrm{~cm})$ e 270 juvenis $(29,6 \pm 0,06 \mathrm{~g}$ e 11,52 $\pm 0,01)$. Os peixes foram mantidos em jejum por 24 horas em quatro caixas de amianto $(500 \mathrm{~L})$ com aeração constante. Em seguida, os peixes foram distribuídos em 54 sacos plásticos, capacidade para 5 L e mantidos em soluções de: cloreto de sódio nas concentrações de ( 0 ; 4; ou $8 \mathrm{~g} / \mathrm{L}$ ), benzocaína $(0 ; 20$; ou $40 \mathrm{mg} / \mathrm{L})$ e óleo de cravo $(0 ; 2 ;$ ou $5 \mathrm{mg} / \mathrm{L})$. O delineamento foi em blocos casualizados, com três repetições, em arranjo multifatorial, com três fatores: substâncias, peixes e concentrações de cada substância em estudo. Mensuraram-se, no início, os parâmetros oxigênio dissolvido, temperatura, pH, salinidade e amônia e, ao final do experimento, a glicose dos peixes. Maiores níveis de glicose foram registrados nos juvenis mantidos na água com óleo de cravo e cloreto de sódio. Não foi observada diferença significativa na sobrevivência entre os tipos de peixes e as substâncias. Entretanto, as dosagens de cloreto de sódio e benzocaína ocasionaram diferenças significativas na taxa de sobrevivência. A sobrevivência foi baixa nos alevinos com $0 \mathrm{~g} / \mathrm{L}$ de cloreto de sódio e diferiu das dosagens 4 e $8 \mathrm{~g} / \mathrm{L}$. O óleo de cravo promoveu resultados similares, em todas as dosagens e substâncias, de taxas de glicose. Ao final do experimento, a sobrevivência foi satisfatória, com média geral de 97,26\%, comprovando que as três substâncias podem ser usadas no transporte de alevinos e juvenis durante 5 horas. Sugere-se o cloreto de sódio por seu melhor custo-benefício.

Palavras-chave: anestésico, estresse, eugenol, glicose, Oreochromis niloticus, sal ( $\mathrm{NaCl})$

\section{Sodium chloride, benzocaine and clove oil in tilapia transport water}

ABSTRACT - The action of different concentrations of sodium chloride, benzocaine and clove oil in the survival of fingerling and juvenile tilapia (Oreochromis niloticus), chitralada lineage, was tested during 5 hours for transportation. A total of 1350 fingerlings were used (weight $9.74 \pm 0.04 \mathrm{~g}$ and length $6.79 \pm 0.01 \mathrm{~cm}$ ) and 270 juveniles (average weight $29.6 \pm 0.06 \mathrm{~g}$ and length $11.52 \pm 0.01 \mathrm{~cm}$ ). The fish were maintained without food for 24 hours in four $500 \mathrm{~L}$ asbestos boxes with constant aeration. Subsequently, the fish were distributed in $545 \mathrm{~L}$ plastic bags and maintained in a solution of sodium chloride at a concentration of $(0 ; 4 ; 8 \mathrm{~g} / \mathrm{L})$, benzocaine $(0 ; 20 ; 40 \mathrm{mg} / \mathrm{L})$ and clove oil $(0 ; 2 ; 5 \mathrm{mg} / \mathrm{L})$. A randomized block experimental design was used with three replications, in multi-factorial arrangements, with three factors: substances, fish and different concentrations for each substance used. Dissolved oxygen, temperature, $\mathrm{pH}$, salinity and ammonia were measured at the beginning of the experiment and the fish glucose at the end of the experiment. The largest glucose levels were detected in the juveniles maintained in water with clove oil and sodium chloride. Significant differences were not observed in the survival among types of fish and substances. However, the doses used of sodium chloride and benzocaine caused significant differences in the survival rate. There was low fingerling survival with $0 \mathrm{~g} / \mathrm{L}$ of sodium chloride that differed from the doses 4 and $8 \mathrm{~g} / \mathrm{L}$. The clove oil presented similar results at all the different doses and all the substances on the glucose rates. Survival was satisfactory at the end of the experiment, with a general average of $97.26 \%$, showing that the three substances can be used to transport fingerlings and juveniles during 5 hours. Sodium chloride is recommended because it was the most cost-effective.

Key Words: anesthetic, eugenol, glucose, Oreochromis niloticus, salt $(\mathrm{NaCl})$, stress 


\section{Introdução}

Entre as espécies de tilápia, a que mais se destaca na piscicultura mundial é a Oreochromis niloticus, por apresentar rusticidade, alto ganho de peso, facilidade de reprodução, excelente sabor da carne e tempo curto para atingir o tamanho comercial (Kubitza, 2000).

Atualmente diversas linhagens de tilápia com excelente desempenho zootécnico estão disponíveis comercialmente, mas uma das mais procuradas no mercado brasileiro é a chitralada (também conhecida como tailandesa), desenvolvida no Japão e melhorada no Palácio Real de Chitralada, na Tailândia (Zimmermann, 2000).

No processo de produção, o transporte de alevinos e juvenis é uma das etapas mais importantes no manejo e na comercialização de peixes. Durante essa prática, os peixes são afetados por uma série de agentes ou fatores estressantes, como captura, superpopulação, mudanças bruscas de temperatura, manuseio, barulho excessivo e o próprio transporte. Segundo Grottum et al. (1997), o sucesso do transporte consiste em conter a maior densidade de peixes no menor volume de água possível, sem que haja mortalidade, deterioração da qualidade da água e estresse. Nesse sentido, são usados produtos condicionadores, como sal marinho e até mesmo alguns anestésicos, para reduzir as agressões causadoras de estresse durante o transporte. Os benefícios do uso do cloreto de sódio na água de transporte de alevinos já foram amplamente documentados (Barton \& Zitzow, 1995; Carneiro \& Urbinati, 2001; Gomes et al., 2003).

Os anestésicos são usados para reduzir a atividade metabólica dos peixes durante o transporte, a pesagem, a marcação e outras manipulações (Gunn, 2000; Pirhonen \& Schreck, 2003). A redução da atividade, do estímulo visual, do consumo de oxigênio e da excreção de amônia pode ser um dos efeitos positivos da utilização de anestésicos (Wurts, 1995). A benzocaína é um dos anestésicos mais utilizados no Brasil em algumas etapas de manejo dos peixes. Trata-se de um anestésico local, cujo potencial de ação é semelhante ao do MS-222 (Mattson \& Ripple, 1989; Burka et al., 1997; Ross \& Ross, 1999). O óleo de cravo é um anestésico utilizado nos peixes em vários países, com vantagens econômicas e sem propriedades tóxicas aparentes (Soto \& Burhanuddin, 1995; Munday \& Wilson, 1997; Sladry et al., 2001; Woody et al., 2002).

Embora existam vários trabalhos científicos sobre o uso dessas substâncias no transporte de peixes, seus efeitos durante o transporte e a manipulação de tilápiasdo-nilo ainda não estão totalmente eluciados. Assim, realizou-se este estudo com o objetivo de avaliar a eficácia de diversas concentrações do condicionador cloreto de sódio e dos anestésicos benzocaína e óleo de cravo-daíndia em reduzir o estresse e aumentar a taxa de sobrevivência de alevinos e juvenis de tilápia (Oreochromis niloticus) durante o transporte.

\section{Material e Métodos}

O experimento foi realizado no período de 11 a 13 de abril de 2007. Foram utilizados 1.350 alevinos com 9,74 $\pm 0,04 \mathrm{~g}$ e $6,79 \pm 0,01 \mathrm{~cm}$ de comprimento e 270 juvenis com $29,6 \pm 0,06 \mathrm{~g}$ e 11,52 $\pm 0,01 \mathrm{~cm}$ de comprimento, ambos de tilápia-do-nilo (Oreochromis niloticus), linhagem chitralada.

$\mathrm{O}$ delineamento experimental utilizado foi em blocos casualizados, com 18 tratamentos em três repetições, em arranjo multifatorial, com três fatores: substâncias, biótipos de peixes (dois) e concentrações por substância (três), em classificação hierárquica parcial. Substâncias e biótipos de peixes (alevinos e juvenis) formaram fatores cruzados e as dosagens estudadas foram aninhadas a cada substância, não se constituindo fatores cruzados. As substâncias foram utilizadas nas seguintes concentrações: cloreto de sódio - 0; 4 ou 8 g/L; benzocaína - 0; 20 ou 40 mg/L; óleo de cravo - $0 ; 2$ ou $5 \mathrm{mg} / \mathrm{L}$.

Depois de capturados, os peixes foram selecionados por tamanho por meio de um classificador modelo D $6002 \mathrm{e}$, em seguida, distribuídos separadamente em quatro caixas de amianto com capacidade para $500 \mathrm{~L}$ de água, com aeração constante, onde foram mantidos em jejum durante 24 horas. Nessa condição, foi realizada amostragem em $10 \%$ dos peixes de cada caixa e aferidos o peso, em balança eletrônica digital de capacidade máxima de $500 \mathrm{~g}$ (precisão de $0,01 \mathrm{~g}$ ), e o comprimento, com auxílio de um ictiômetro.

Os peixes foram acondicionados em sacos plásticos transparentes com capacidade para $20 \mathrm{~L}$ contendo $5 \mathrm{~L}$ de água e as substâncias em estudo. Cada unidade experimental foi constituída de um saco plástico contendo 50 alevinos ou 10 juvenis. A benzocaína foi previamente diluída em etanol na proporção de 1:10, enquanto o óleo de cravo foi diluído em etanol na proporção de 1:20.

Os peixes foram mantidos dentro dos sacos no laboratório durante 5 horas, tempo médio utilizado no transporte de alevinos e juvenis. O grau de interferência dos anestésicos (benzocaína e óleo de cravo) no comportamento dos peixes foi registrado segundo Roubach \& Gomes (2001) e Woody et al. (2002) (Tabela 1).

No início e ao final de cada ensaio, foram mensuradas as seguintes variáveis da água: oxigênio dissolvido (mg/L) 
Tabela 1 - Características comportamentais de alevinos e juvenis de tilápia-do-nilo expostos aos anestésicos benzocaína e óleo de cravo

\begin{tabular}{|c|c|c|}
\hline \multirow[t]{2}{*}{ Estágio } & \multicolumn{2}{|c|}{ Descrição do comportamento } \\
\hline & Roubach \& Gomes (2001) & Woody et al. (2002) \\
\hline 1 & $\begin{array}{l}\text { Sedação leve. Perda de reação } \\
\text { aos movimentos visuais e ao toque }\end{array}$ & Desbalanço visível dos movimentos operculares \\
\hline 2 & Anestesia leve. Perda parcial do equilíbrio & $\begin{array}{l}\text { Perda parcial de equilíbrio e dificuldade em } \\
\text { manter posição normal de nado, quando parado }\end{array}$ \\
\hline 3 & Anestesia profunda. Perda total do equilíbrio & $\begin{array}{l}\text { Perda total de equilíbrio e incapacidade } \\
\text { de recuperar a posição vertical de nado }\end{array}$ \\
\hline 4 & $\begin{array}{c}\text { Anestesia cirúrgica I. Diminuição dos } \\
\text { movimentos operculares }\end{array}$ & Ausência de reação a qualquer estímulo \\
\hline 5 & Anestesia cirúrgica II. Mínimo movimento opercular & $\begin{array}{l}\text { Recuperação da posição normal } \\
\text { de nado e da capacidade de nadar }\end{array}$ \\
\hline 6 & Colapso medular. Overdose & \\
\hline
\end{tabular}

e temperatura $\left({ }^{\circ} \mathrm{C}\right)$, com auxílio de um oxímetro YSI modelo 550-a; pH, com peagâmetro marca intelligent metter; salinidade (UPS), mensurada com multiparâmetro YSI 556; e amônia total $(\mathrm{mg} / \mathrm{L})$, determinada no Laboratório de Limnologia da Universidade Federal Rural de Pernambuco -UFRPE.

Ao final do experimento, coletou-se sangue com seringa de $3 \mathrm{~mL}$ heparinizada, de 27 alevinos e 27 juvenis (de cada saco nas três repetições) e, utilizando-se um medidor digital, marca Accu-Chek (Active), determinou-se a glicose, em mg/dL. Logo em seguida, foram registrados 19 alevinos e 11 juvenis mortos. Os sobreviventes foram liberados no viveiro e 24 horas depois, mais uma vez, observou-se o índice de mortalidade, quando se registraram 12 alevinos e 4 juvenis mortos.

Foram realizadas análises de correlação das variáveis: glicose, amônia, salinidade e comprimento do peixe sobre a mortalidade dos peixes, utilizando-se o modelo estatístico:

$$
\mathrm{Y}_{\mathrm{ijkl}}=\mathrm{M}+\mathrm{P}_{\mathrm{i}}+\mathrm{S}_{\mathrm{j}}+(\mathrm{PS})_{\mathrm{ij}}+\mathrm{S} / \mathrm{D}_{\mathrm{ik}}+(\mathrm{PS})_{\mathrm{ij}} / \mathrm{D}_{\mathrm{k}}+\varepsilon_{\mathrm{ijkl}} \text {, }
$$

em que: $Y_{\mathrm{ijkl}}=$ observação na parcela do l-ésimo blocos referentes aos n tratamentos $(1=1,2$ e 3$) ; \mathrm{M}=$ média geral do experimento; $\mathrm{P}_{\mathrm{i}}=$ efeito do i-ésimo biótipo de peixes ( $i=1$ e 2 , ou seja, alevinos e juvenis); $S_{j}=$ efeito da j-ésima substância ( j = 1,2 e 3, ou seja, cloreto de sódio, benzocaína e óleo de cravo); (PS $)_{\mathrm{ij}}=$ efeito da interação biótipo de peixes (i) $\times$ substância $(\mathrm{j}) ; S / \mathrm{D}_{\mathrm{jk}}=$ efeito da k-ésima dosagem dentro de cada substância (j); (PS) $)_{\mathrm{ij}} / \mathrm{D}_{\mathrm{k}}=$ efeito da dosagem (k) dentro da interação (PS $)_{\mathrm{ij}} ; \varepsilon_{\mathrm{jkl}}=$ efeito de erros aleatórios, independentes e normalmente distribuídos com média (zero) e variância $\sigma^{2}$, associados à observação (ijk) (Euclydes, 1983).

\section{Resultados e Discussão}

A temperatura média da água nos períodos inicial e final, para alevinos e juvenis, manteve-se em torno de 28,32 e $29,55^{\circ} \mathrm{C}$, respectivamente, e esses valores estão dentro do conforto térmico $\left(27 \mathrm{a} 30^{\circ} \mathrm{C}\right)$ para a espécie (Kubitza, 2000). Não houve diferença $(\mathrm{P}>0,05)$ na temperatura da água durante o período experimental.

A concentração de oxigênio dissolvido da água inicial e final variou entre 1,98 e $18,99 \mathrm{mg} / \mathrm{L}$ alevinos e juvenis. Segundo Kubitza (2000), quando a concentração de oxigênio dissolvido atinge 45 a $50 \%$ da saturação (aproximadamente 3 a $3,5 \mathrm{mg} / \mathrm{L}$, a $28-30^{\circ} \mathrm{C}$ ), a tilápia começa a reduzir sua atividade. Nesta pesquisa, as substâncias estressantes foram adicionadas na água antes da injeção do oxigênio. Assim, já no início dos tratamentos, as substâncias já promoviam redução no metabolismo.

Neste experimento, as altas concentrações de oxigênio dissolvido no final (acima de $100 \%$ de saturação) provavelmente estão relacionadas à supersaturação na injeção do oxigênio nos sacos plásticos no início da investigação. Adicionar oxigênio nos sacos plásticos usados para o transporte é um processo rotineiro nas estações de piscicultura, pois favorece o acondicionamento dos alevinos para manter os níveis de oxigênio sempre elevados.

O pH da água inicial e final variou de 5,71 a 6,30, respectivamente, para alevinos e juvenis (Tabela 2) e encontra-se no limite desejável, pois a faixa ideal para a espécie, segundo Kubitza (2000), é de 6,0 a 8,5.

A variação de nitrogênio amoniacal total, inicial e final foi 0,05 e 3,59 mg/L, respectivamente, para alevinos e juvenis. A concentração de amônia total final diferiu $(\mathrm{P}<0,05)$ entre 
Tabela 2 - Qualidade da água em cada tratamento (tamanho de peixe e substâncias condicionantes)

\begin{tabular}{|c|c|c|c|c|c|c|c|c|}
\hline \multirow[t]{3}{*}{ Tratamento } & \multicolumn{8}{|c|}{ Parâmetro físico e químico } \\
\hline & \multicolumn{2}{|c|}{ Temperatura $\left({ }^{\circ} \mathrm{C}\right)$} & \multicolumn{2}{|c|}{ Oxigênio dissolvido ( $\mathrm{mg} / \mathrm{L})$} & \multicolumn{2}{|c|}{$\mathrm{pH}$} & \multicolumn{2}{|c|}{ Amônia total $(\mathrm{mg} / \mathrm{L})$} \\
\hline & Inicial & Final & Inicial & Final & Inicial & Final & Inicial & Final \\
\hline \multicolumn{9}{|l|}{ Tamanho dos peixes } \\
\hline Alevinos & $28,49 a$ & $29,61 \mathrm{a}$ & $3,16 \mathrm{a}$ & $17,33 b$ & $5,84 a$ & $6,19 \mathrm{a}$ & $0,05 \mathrm{a}$ & $3,59 \mathrm{a}$ \\
\hline Juvenis & $28,16 \mathrm{a}$ & $29,49 a$ & $3,09 \mathrm{a}$ & $18,99 \mathrm{a}$ & $5,89 \mathrm{a}$ & $6,23 \mathrm{a}$ & $0,05 \mathrm{a}$ & $2,59 \mathrm{~b}$ \\
\hline \multicolumn{9}{|l|}{ Substância } \\
\hline Cloreto de sódio & $28,55 \mathrm{a}$ & $29,56 \mathrm{a}$ & $3,39 \mathrm{a}$ & $18,49 \mathrm{a}$ & $5,77 \mathrm{~b}$ & $6,00 \mathrm{~b}$ & $0,05 \mathrm{a}$ & $3,13 \mathrm{a}$ \\
\hline Benzocaína & $28,22 \mathrm{a}$ & $29,54 \mathrm{a}$ & $3,15 \mathrm{a}$ & $18,02 \mathrm{a}$ & $5,89 \mathrm{ab}$ & $6,26 \mathrm{a}$ & $0,05 \mathrm{a}$ & $2,97 \mathrm{a}$ \\
\hline Óleo de cravo & $28,20 \mathrm{a}$ & $29,56 \mathrm{a}$ & $2,84 \mathrm{a}$ & $17,99 \mathrm{a}$ & $5,93 \mathrm{a}$ & $6,28 \mathrm{a}$ & $0,05 \mathrm{a}$ & $3,18 \mathrm{a}$ \\
\hline \multicolumn{9}{|c|}{ Dosagem $(\mathrm{g} / \mathrm{L}), \mathrm{NaCl}$} \\
\hline 0 & $28,52 \mathrm{a}$ & $29,77 \mathrm{a}$ & $4,48 \mathrm{a}$ & $20,40 \mathrm{a}$ & $5,81 \mathrm{a}$ & $6,26 \mathrm{a}$ & $0,05 \mathrm{a}$ & $3,15 \mathrm{a}$ \\
\hline 4 & $28,62 \mathrm{a}$ & $29,42 \mathrm{a}$ & $2,60 \mathrm{~b}$ & $17,78 \mathrm{~b}$ & $5,79 \mathrm{a}$ & $6,01 \mathrm{~b}$ & $0,05 \mathrm{a}$ & $2,85 \mathrm{a}$ \\
\hline 8 & $28,52 \mathrm{a}$ & $29,48 \mathrm{a}$ & $3,08 \mathrm{~b}$ & $17,30 \mathrm{~b}$ & $5,71 \mathrm{a}$ & $5,98 \mathrm{~b}$ & $0,05 \mathrm{a}$ & $3,40 \mathrm{a}$ \\
\hline \multicolumn{9}{|c|}{ Dosagem (mg/L), benzocaína } \\
\hline 0 & $28,25 \mathrm{a}$ & $29,60 \mathrm{a}$ & $4,32 \mathrm{a}$ & $17,68 \mathrm{a}$ & $5,75 \mathrm{~b}$ & $6,25 \mathrm{a}$ & $0,05 \mathrm{a}$ & $3,28 \mathrm{a}$ \\
\hline 20 & $28,12 \mathrm{a}$ & $29,45 \mathrm{a}$ & $2,77 b$ & $18,08 \mathrm{a}$ & $6,08 \mathrm{a}$ & $6,27 \mathrm{a}$ & $0,05 \mathrm{a}$ & $2,88 \mathrm{a}$ \\
\hline 40 & $28,30 \mathrm{a}$ & $29,57 \mathrm{a}$ & $2,37 \mathrm{~b}$ & $18,28 \mathrm{a}$ & $5,86 a b$ & $6,27 \mathrm{a}$ & $0,05 \mathrm{a}$ & $2,77 \mathrm{a}$ \\
\hline \multicolumn{9}{|c|}{ Dosagem $(\mathrm{mg} / \mathrm{L})$, óleo de cravo } \\
\hline 0 & $26,37 \mathrm{a}$ & $29,82 \mathrm{a}$ & $4,47 \mathrm{a}$ & $17,13 \mathrm{a}$ & $5,80 \mathrm{a}$ & $6,27 \mathrm{a}$ & $0,05 \mathrm{a}$ & $3,19 \mathrm{a}$ \\
\hline 2 & $28,12 \mathrm{a}$ & $29,43 \mathrm{a}$ & $1,98 b$ & $18,45 \mathrm{a}$ & $5,98 \mathrm{a}$ & $6,28 \mathrm{a}$ & $0,05 \mathrm{a}$ & $3,30 \mathrm{a}$ \\
\hline 5 & $28,12 \mathrm{a}$ & $29,43 \mathrm{a}$ & $2,07 \mathrm{~b}$ & $18,38 \mathrm{a}$ & $6,01 \mathrm{a}$ & $6,30 \mathrm{a}$ & $0,05 \mathrm{a}$ & $3,04 \mathrm{a}$ \\
\hline Média & 28,32 & 29,55 & 3,12 & 18,17 & 5,87 & 6,21 & 0,05 & 3,09 \\
\hline CV (\%) & 2,15 & 1,07 & 28,38 & 9,09 & 3,02 & 1,69 & 0,00 & 15,66 \\
\hline
\end{tabular}

Letras diferentes na mesma coluna diferem $(\mathrm{P}<0,05)$ pelo teste Tukey.

alevinos (3,59 mg/L) e juvenis (2,59 mg/L), o que pode estar relacionado à elevada densidade dos alevinos, que aumenta o processo de excreção nitrogenada. Concentrações letais de amônia, que matam 50\% dos animais em 24, 48 e 96 horas, foram determinadas para tilápias-vermelhas híbridas (O. niloticus $\times$ O. mossambicus), em torno de 6,6; 4,0 e 2,6 mg/L, respectivamente (Daud et al., 1988). Considerando que o tempo médio para o transporte de alevinos e juvenis foi de 5 horas, a concentração de amônia encontrada neste experimento não foi letal para tilápia (Oreochromis niloticus).

No tratamento com benzocaína na dosagem de $40 \mathrm{mg} / \mathrm{L}$, após 5 minutos de exposição à substância, 100\% dos juvenis de tilápia apresentaram-se no estágio 3 (Tabela 1), ou seja, sedados sem locomoção. Barbosa et al. (2006) verificaram que o uso de 0,05 g/L de benzocaína resultou em maior tempo para que juvenis de pacu (Piaractus mesopotamicus) atingissem o estágio de anestesia, enquanto, na mesma dosagem, aproximadamente $80 \%$ dos alevinos permaneceram sedados durante os primeiros 60 minutos.

Após os 120 minutos de exposição a $40 \mathrm{mg} / \mathrm{L}$ de benzocaína, 30 e $80 \%$ dos juvenis e alevinos, respectiva- mente, apresentaram recuperação e capacidade natatória, estágio 5 (Tabela 1). No final dos 180 minutos de exposição a $40 \mathrm{mg} / \mathrm{L}$ de benzocaína, apenas 4 e $2 \%$ de juvenis e alevinos, respectivamente, permaneceram no estágio $2 \mathrm{e}$, ao final dos 240 minutos de exposição a $40 \mathrm{mg} / \mathrm{L}$ de benzocaína, $100 \%$ de juvenis e alevinos demonstraram comportamento natatório normal. Na dosagem de $20 \mathrm{mg} / \mathrm{L}$ de benzocaína, após 5 minutos de exposição à substância, $45 \%$ da população dos alevinos e juvenis de tilápia (Oreochromis niloticus) apresentou-se inativa, enquanto, durante 180 minutos, apenas 3\% dos peixes apresentavam-se em inatividade. Após 240 minutos, 100\% dos alevinos e juvenis já apresentavam capacidade natatória normal.

Nas dosagens utilizadas, o uso do cloreto de sódio não alterou o comportamento dos peixes, que permaneceram com natação normal após 240 minutos de exposição. Carneiro \& Urbinati (2001) sugeriram que a adição de 6 g/L de cloreto de sódio na água de transporte reduziu as alterações fisiológicas de estresse em matrinxã.

No tratamento com óleo de cravo na dosagem de $5 \mathrm{mg} / \mathrm{L}$ após 60 minutos de exposição, 40 e $20 \%$ de juvenis e alevinos, respectivamente, apresentaram-se no estágio 2 
(Tabela 1). Após 240 minutos de exposição a essa substância, alevinos e juvenis apresentaram comportamento normal de natação. Com $2 \mathrm{mg} / \mathrm{L}$ de óleo de cravo, alevinos e juvenis voltaram à capacidade natatória normal durante todo o experimento.

Nos tratamentos sem substâncias (controle), os alevinos e juvenis apresentaram comportamento de natação regular até 240 minutos de estudo. A taxa de mortalidade dos peixes, em todos os tratamentos, foi registrada após 240 minutos de experimento, e apresentou valor médio de sobrevivência de $97,26 \%$.

Após o período de exposição dos peixes às substâncias, a maior média de glicose no sangue dos peixes entre os tratamentos foi registrada nos juvenis $(53,74 \mathrm{mg} / \mathrm{dL}) \mathrm{em}$ relação à média observada nos alevinos $(47,37 \mathrm{mg} / \mathrm{dL}) \mathrm{de}$ tilápia, Oreochromis niloticus (Tabela 3). Os maiores teores de glicose foram observados nos tratamentos com benzocaína nos alevinos e juvenis.

Os teores médios de glicose no sangue (Figura 1) foram 47,05; 59,50; e 45,11 mg/dL nos tratamentos com cloreto de sódio, benzocaína e óleo de cravo, respectivamente, que não diferiram $(\mathrm{P}>0,05)$ entre si.

Tabela 3 - Valores médios de glicose, peso e comprimento e taxa de sobrevivência de alevinos e juvenis de tilápia-donilo (Oreochromis niloticus)

\begin{tabular}{|c|c|c|c|c|}
\hline Tratamento & $\begin{array}{l}\text { Glicose } \\
(\mathrm{mg} / \mathrm{dL})\end{array}$ & $\begin{array}{c}\text { Peso } \\
(\mathrm{g})\end{array}$ & $\begin{array}{l}\text { Comprimento } \\
(\mathrm{cm})\end{array}$ & $\begin{array}{c}\text { Sobrevivência } \\
(\%)\end{array}$ \\
\hline \multicolumn{5}{|c|}{ Tamanho dos peixes } \\
\hline Alevinos & $47,37 \mathrm{a}$ & $9,37 \mathrm{~b}$ & $6,75 b$ & $98,59 a$ \\
\hline Juvenis & $53,74 \mathrm{a}$ & $29,67 \mathrm{a}$ & $11,52 \mathrm{a}$ & $95,93 \mathrm{a}$ \\
\hline \multicolumn{5}{|l|}{ Substâncias } \\
\hline Cloreto de sódi & $47,05 \mathrm{a}$ & $19,83 \mathrm{a}$ & $9,13 \mathrm{a}$ & $96,22 \mathrm{a}$ \\
\hline Benzocaína & $59,50 \mathrm{a}$ & $18,33 \mathrm{a}$ & $8,97 \mathrm{a}$ & $96,56 a$ \\
\hline Óleo de cravo & $45,11 \mathrm{a}$ & $20,39 a$ & $9,32 \mathrm{a}$ & $99,00 \mathrm{a}$ \\
\hline
\end{tabular}

Dosagens $(\mathrm{g} / \mathrm{L}), \mathrm{NaCl}$

$\begin{array}{llllr}0 & 39,50 \mathrm{a} & 17,00 \mathrm{~b} & 8,67 \mathrm{a} & 88,67 \mathrm{~b} \\ 4 & 45,83 \mathrm{a} & 18,33 \mathrm{ab} & 8,73 \mathrm{a} & 100,00 \mathrm{a} \\ 8 & 55,83 \mathrm{a} & 24,17 \mathrm{a} & 9,98 \mathrm{a} & 100,00 \mathrm{a}\end{array}$

Dosagens $(\mathrm{mg} / \mathrm{L})$, benzocaína

$\begin{array}{llllr}0 & 35,67 \mathrm{~b} & 21,17 \mathrm{a} & 8,92 \mathrm{a} & 100,00 \mathrm{a} \\ 20 & 46,00 \mathrm{~b} & 16,17 \mathrm{a} & 8,90 \mathrm{a} & 100,00 \mathrm{a} \\ 40 & 96,83 \mathrm{a} & 17,67 \mathrm{a} & 9,10 \mathrm{a} & 89,67 \mathrm{~b}\end{array}$

Dosagens (mg/L), Óleo de cravo

\begin{tabular}{ccccc}
0 & $42,50 \mathrm{a}$ & $15,83 \mathrm{~b}$ & $8,88 \mathrm{a}$ & $97,67 \mathrm{a}$ \\
2 & $36,33 \mathrm{a}$ & $24,17 \mathrm{a}$ & $9,33 \mathrm{a}$ & $100,00 \mathrm{a}$ \\
5 & $55,50 \mathrm{a}$ & $21,17 \mathrm{ab}$ & $9,73 \mathrm{a}$ & $99,33 \mathrm{a}$ \\
\hline Média & 50,56 & 19,52 & 9,14 & 97,26 \\
CV $(\%)$ & 43,04 & 23,28 & 11,34 & 2,42 \\
\hline
\end{tabular}

Letras diferentes na mesma coluna diferem $(\mathrm{P}<0,05)$ pelo teste Tukey.

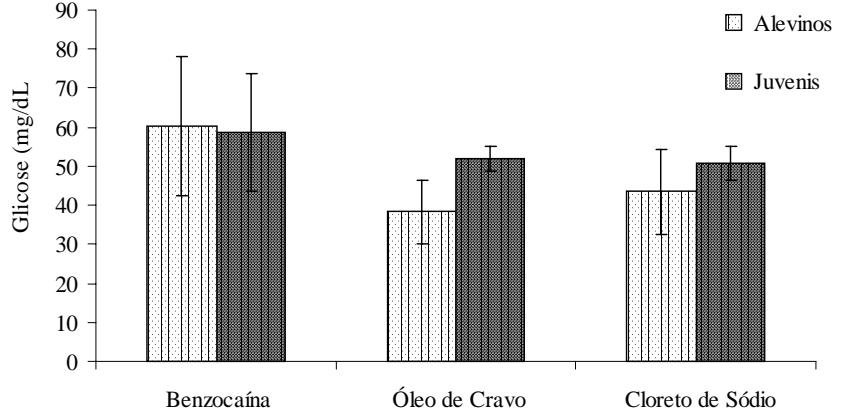

Figura 1 - Teores de glicose no sangue no sangue de alevinos e juvenis de tilápia-do-nilo (Oreochromis niloticus) mantidos em água com benzocaína, óleo de cravo e cloreto de sódio (mg/dL).

Os indicadores mais utilizados para avaliação do estresse são o cortisol e a glicose. O cortisol caracteriza a resposta primária e a glicose a resposta secundária do estresse (Robertson et al., 1987). A glicemia, uma das respostas fisiológicas mais utilizadas como indicador de estresse em peixes, aumenta na presença de algum fator estressante para suprir a maior demanda energética, característica de situações desfavoráveis (Morgan \& Iwama, 1997).

Os teores de glicose no sangue $(\mathrm{mg} / \mathrm{dL})$ diferiram $(\mathrm{P}<0,05)$ entre as dosagens de benzocaína e foram maiores na dosagem de $40 \mathrm{mg} / \mathrm{L}$ (Tabela 3 ). Correlação positiva entre o aumento das dosagens de benzocaína e o teor de glicose no sangue foi visualizada e ajustou-se a uma equação linear expressa por: Glicose $(\mathrm{mg} / \mathrm{dL})=28,92+1,5922$ (benzocaína, $\mathrm{mg} / \mathrm{L}) ; \mathrm{r}^{2}=0,8725$.

Carneiro et al. (2002) estudaram o transporte de matrinxã, Brycon cephalus e também observaram que o uso de benzocaína contribuiu para elevação da glicemia e dos níveis plasmáticos do cortisol. Esses autores registraram efeitos negativos das concentrações mais altas (10 e $20 \mathrm{mg} / \mathrm{L}$ ) e concluíram que essa substância não reduziu o estresse causado pelo transporte. Gomes et al. (2001) testaram diversas concentrações de benzocaína em juvenis de Colossoma macropomum e observaram que, nos peixes expostos a altas doses (200 - $350 \mathrm{mg} / \mathrm{L})$, houve aumento significativo do nível de glicose.

Neste trabalho a ausência de cloreto de sódio resultou em baixos valores de sobrevivência $(88,67 \%)$, diferindo significativamente das dosagens 4 e $8 \mathrm{~g} / \mathrm{L}$, que resultaram em $100 \%$ de sobrevivência $(\mathrm{P}<0,05)$. Alevinos e juvenis não foram afetados pela interferência da salinidade. A adição de cloreto de sódio à água tem sido utilizada para mitigação de estresse e aumento da taxa de sobrevivência no transporte de matrinxã (Brycon cephalus), de acordo com Urbinati \& Carneiro (2001). 
Entretanto, houve resposta altamente significativa à dosagem, independentemente do tamanho do peixe, cuja análise de regressão para as dosagens 0,4 e 8 g/L de cloreto de sódio comprovou resposta linear, representada pela equação: $\mathrm{Y}=0,306+0,66041, \mathrm{P}<0,00001$, com coeficiente determinação $\left(\mathrm{r}^{2}\right)=0,9996$. A taxa de crescimento de salinidade foi em torno de $0,66 \mathrm{~g} / \mathrm{L}$ para cada unidade de cloreto de sódio aplicada. Existe correlação positiva de 0,7175 entre os níveis de $\mathrm{NaCl}$ e sobrevivência dos peixes, ou seja, o aumento na taxa de $\mathrm{NaCl}$ no limite estudado elevou a taxa de sobrevivência.

Neste estudo, o óleo de cravo promoveu resultados similares, tanto entre dosagens quanto entre substâncias, de glicose no sangue. Segundo Anderson et al. (1997), o óleo de cravo é mais efetivo como anestésico em concentrações de 40-60 mg/L para salmonídeos. Sladky et al. (2001) observaram aumento das concentrações de glicose em pacus (Piaractus brachypomus) anestesiados com MS-222 e óleo de cravo, contudo, esses pesquisadores não relataram diferença significativa nos níveis de glicose entre os anestésicos.

Resultados de sobrevivência satisfatórios foram registrados durante o período experimental, com média geral de 97,26\% (Tabela 3). Não foi observada diferença significativa $(\mathrm{P}>0,05)$ na taxa de sobrevivência entre os tamanhos dos peixes e as substâncias utilizadas. No entanto, as dosagens de cloreto de sódio e benzocaína ocasionaram diferença significativa $(\mathrm{P}<0,05)$ na taxa sobrevivência (Tabela 3 ).

De acordo com a equação de regressão quadrática da sobrevivência em relação à substância $\mathrm{NaCl}$, em que Sobrevivência $(\%)=88,67+4,2499(\mathrm{NaCl}$ g/L $)-0,0354$ $(\mathrm{NaCl} g / \mathrm{L})^{2} ; \mathrm{r}^{2}=1$, sugere-se a dosagem de $6 \mathrm{~g} / \mathrm{L}$ de cloreto de sódio para aumentar a sobrevivência durante o transporte

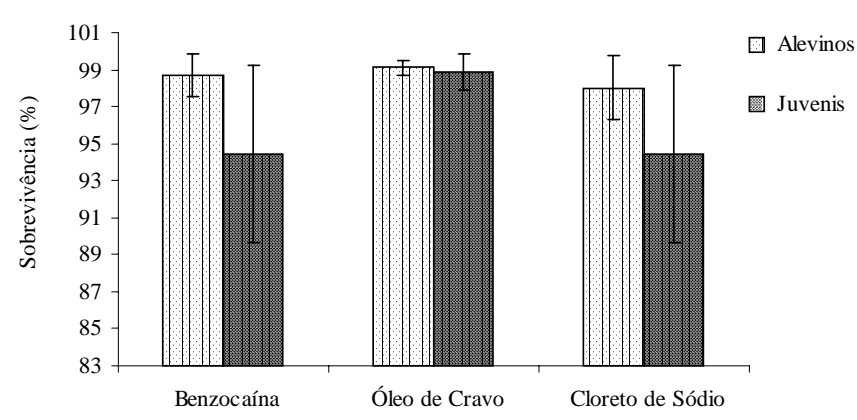

Figura 2 - Sobrevivência de alevinos e juvenis de tilápia (Oreochromis niloticus) mantidos em água com as substâncias benzocaína, óleo de cravo e cloreto de sódio. de tilápias. No caso da benzocaína, recomenda-se utilizar $20 \mathrm{mg} / \mathrm{L}$ de água, na concentração de $30 \mathrm{mg} / \mathrm{L}$, como apresentado na equação de regressão quadrática: Sobrevivência $(\%)=89,67+0,7750\left(\right.$ Benzocaínamg/L) $-0,0129(\text { Benz.mg/L })^{2}$; $\mathrm{r}^{2}=1$. A concentração de $40 \mathrm{mg} / \mathrm{L}$ de benzocaína, no entanto, interferiu negativamente na sobrevivência dos alevinos e juvenis de tilápias (Tabela 3 ).

Os resultados da influência das três substâncias na sobrevivência dos peixes (Figura 2), cujos valores médios foram de 96,22; 96,56 e 99,00\% para cloreto de sódio, benzocaína e óleo de cravo, respectivamente, não diferiram $(\mathrm{P}>0,05)$ significativamente entre as substâncias.

\section{Conclusões}

Ao final do experimento, a sobrevivência teve resultado satisfatório, com média geral de 97,26\%, comprovando que as três substâncias podem ser usadas no transporte de alevinos e juvenis durante 5 horas. O cloreto de sódio pode ser utilizado nas concentrações de 6 a $8 \mathrm{~g} / \mathrm{L}$, uma vez que tem custo inferior ao da benzocaína e ao do óleo de cravo, é de fácil aquisição, tem efeito antisséptico e ainda reduz o estresse de tilápias-do-nilo (Oreochromis niloticus).

\section{Literatura Citada}

ANDERSON, W.G.; McKINLEY, R.S.; COLVECCHIA, M. The use of clove oil as an anaesthetic for rainbow trout and its effects on swimming performance North American Journal of Fisheries Management, v.17, p.301-307, 1997.

BARBOSA, A.L.F.; TAKATA, R.; PORTELlA, M.C. et al. Respostas de juvenis de pacu Piaractus mesopotamicus à anestesia com diferentes concentrações de benzocaína. In: AquaCiência 2006, Bento Gonçalves. Anais... Bento Gonçalves, 2006. (CD-ROM).

BARTON, B.A.; ZITZOW, R.E. Physiological responses of juvenile walleyes to hading stress with recovery in saline water, Progressive Fish-Culturist, v.57, p.267-276, 1995.

BURKA, J.F.; HAMMELL, K.L.; HORSBERG, T.E. et al. Drugs in salmonid aquaculture - a review. Journal of Veterinary Pharmacology and Therapeutics, v.20, p.333-349, 1997.

CARNEIRO, P.C.F.; URBINATI, E.C. Salt as a stress response mitigator of matrinxã Brycon cephalus (Günther, 1869) during transport. Aquaculture Research, v.32, p.297-304, 2001 .

CARNEIRO, P.C.F.; MARTINS, M.L.; URBINATI, E.C. et al. Transport with different benzocaine concentrations and its consequences on hematological parameters and gill parasite population of matrinxã Brycon cephalus (Günther, 1869) (Osteichthyes, Characidae). Acta Scientiarum, v.24, n.2, p.555-560, 2002 .

DAUD, S.K.; HASBOLLAH, D.; LAW, A.T. Effects of unionized ammonia on red tilapia (Oreochromis mossambicus/ O. niloticus hybrid) fry. In: INTERNATIONAL SYMPOSIUM ON TILAPIA IN AQUACULTURE, 2., 1988, [S.L.]. Proceedings... [S.L.]: ICLARM, 1988. p.411-413.

EUCLYDES, R.F. Manual de utilização do programa SAEG (Sistemas para Análises Estatísticas e Genéticas). Viçosa, MG: Universidade Federal de Viçosa, 1983. 59p. 
GOMES, L.C.; CHIPPARI-GOMES, A.R.; LOPES, N.P. et al. Efficacy of benzocaine as an anesthetic in juvenile tambaqui Colossoma macropomum. Journal of the World Aquaculture Society, v.32, p.426-431, 2001.

GOMES, L.C.; ARAUJO-LIMA, C.A.; ROUBACH, R. et al. Avaliação dos efeitos do sal e da densidade no transporte de tambaqui. Pesquisa Agropecuária Brasileira, v.38, p.283-290, 2003.

GROTTUM, J.A.; STAURNES, M.; SIGHOLT, T. Effect of oxygenation, aeration and $\mathrm{pH}$ control on water quality and survival of turbot, Scophthalmus maximus (L.), kept at high densities during transport. Aquaculture Research, v.28, n.2, p.159-164, 1997.

GUNN, E. [2000]. Floundering in the foibles of fish anesthesia. [S.L.]: Universidade do Algarve. Disponível em: <http://www.ualg.pt/npfcma/docs/trab_bmp/taq_anestesia.pdf>. Acesso em: 22/12/2006.

KUBITZA, F. Tilápia: tecnologia e planejamento na produção comercial. Jundiaí: [s.n.], 2000.

MATTSON, N.S.; RIPPLE, T.H. Metomidate, a better anesthetic for cod (Gadus morhua) in comparison with benzocaine, MS-222, chlorobutanol, and phenoxyethanol. Aquaculture, v.83, p.89-94, 1989.

MORGAN, J.D.; IWAMA, G.K. Measurements of stressed states in the field. In: IWANA, G.W.; PICKERING, A.D.; SUMPTER, J.P. (Eds.). Fish stress and health in aquaculture. Cambridge: University Press, 1997. p.247-270.

MUNDAY, P.L.; WILSON, S.K. Comparative efficacy of clove oil and other chemicals in anaesthetization of Pomacentrus amboinensis, a coral reef fish. Journal Fish Biology, v.51, p.931-938, 1997.
PIRHONEN, J.; SCHRECK, C.B. Effects of anesthesia with MS222, clove oil and $\mathrm{CO}_{2}$ on feed intake and plasma cortisol in steelhead trout (Oncorhynchus mykiss). Aquaculture, v.220, p.507-514, 2003.

ROBERTSON, L.; THOMAS, P.; ARNOLD, C.R. et al. Plasma cortisol and secondary stress responses of red drum to handling, transport, rearing density, and a disease outbreak. Progressive Fish-Culturist, v.49, n.1, p.1-12, 1987.

ROSS, L.G.; ROSS, B. Anaesthetic and sedative techniques for aquatic animals. 2.ed. London: Blackwell, 1999. p.159.

ROUBACH, R.; GOMES, L.C. O uso de anestésicos durante o manejo de peixes. Panorama da Aqüicultura, v.11, n.66, p.37-40, 2001.

SLADKY, K.K.; SWANSON, C.R.; STOSKOPF, M.K. et al. Comparative efficacy of tricaine methanesulfonate and clove oil for use as anesthetics in red pacu (Piaractus brachypomus). American Journal Veterinary Research, v.62, n.3, p.337-342, 2001.

SOTO, C.; BURHANUDDIN. Clove oil as a fish anaesthetic for measuring length and weight of rabitfish (Siganus lineatus). Aquaculture, v.136, p.149-152, 1995.

URBINATI, E.C.; CARNEIRO, P.C.F. Metabolic and hormonal responses of matrinxã, Brycon cephalus, (Teleost: Characidae) to transport stress under influence of benzocaine. Journal of Aquaculture in the Tropics, v.16, n.1, p.75-85, 2001.

WOODY, C.A.; NELSON, J.; RAMSTAD, K. [2002]. Clove oil as an anaesthetic for adultsockeye salmon: field trails. Disponível em: <http://www.idealibrary.com>. Acesso em: 5/5/2007.

WURTS, W.A. Using salt to reduce handling stress in channel catfish. World Aquaculture, v.26, p.80-81, 1995.

ZIMMERMANN, S. O bom desempenho das chitraladas no Brasil. Panorama da Aqüicultura, v, 10, n.60, p.15-19, 2000. 